\title{
Asymmetries in Structure Factor Histograms
}

\author{
G. Korniss, B. Schmittmann and R. K. P. Zia \\ Center for Stochastic Processes in Science and Engineering \\ and \\ Department of Physics, Virginia Polytechnic Institute and State \\ University \\ Blacksburg, Virginia 24061-0435, USA
}

(February 26, 1997)

\begin{abstract}
We investigate the dynamics of a three-state stochastic lattice gas, consisting of holes and two oppositely "charged" species of particles, under the influence of an "electric" field, at zero total charge. Interacting only through an excluded volume constraint, particles can hop to nearest neighbour empty sites. Using a combination of Langevin equations and Monte Carlo simulations, we study the probability distributions of the steady-state structure factors in the disordered phase where homogeneous configurations are stable against small harmonic perturbations. Simulation and theoretical results are in excellent agreement, both showing universal asymmetric exponential distributions.
\end{abstract}

PACS: $64.60 \mathrm{Cn}, 66.30 \mathrm{Hs}, 82.20 \mathrm{Mj}$

Keywords: Non-equilibrium lattice gas; Monte Carlo simulations; Langevin equations; Probability distributions

\section{INTRODUCTION}

Analyzing structure factors and correlations in many-particle systems is a standard way to study collective behaviour in real experiments, computer simulations and theoretical frameworks. For example, for a system with short range microscopic interactions, placed in thermal equilibrium, there are no long range spatial correlations in general. Their presence is a typical signal that the system is at a critical point. On the other hand, when such systems are driven into non-equilibrium steady states, long range correlations are often observed [1]. Another good example is the driven Ising lattice gas [2]3. The role of the drive ("external field") is to bias hopping rates along a particular direction on the lattice. In addition to many other unexpected features such as non-Hamiltonian fixed points controlling the critical behavior 沟, the system exhibits long range spatial correlations at all temperatures above criticality, as a result of the breakdown of the traditional fluctuation-dissipation relations [5]. In momentum space, this appears as a discontinuity singularity of the structure factor at the origin [6].

In this Paper, we revisit a simple, non-equilibrium model [7] displaying similar features and examine the steady state structure factors from a more general point of view. In particular, we will construct the full distribution of various structure factors, using both Monte Carlo simulations and Langevin equations following the method in ref [8]. The model can be considered as a generalization of the KLS [2] model, in a fashion similar to an equilibrium Ising model being extended to spin-1 [9] or Potts [10] models, in that it involves two species of particles (and holes). Referred to as +'s and -'s, they are driven in opposite directions, subject to periodic boundary conditions. To keep the model simple, we suppress the usual Ising nearest neighbor interaction and retain only the excluded volume constraint and the bias. In the simplest scenario, charge exchange $(+\leftrightarrow-)$ is not allowed. Phase transitions in this multi-species model, in both one and two dimensions, have been investigated extensively [11 [16]. Monte Carlo simulations [11] in two dimensions and mean-field studies 12, 13] show that there is a transition, controlled by particle density and drive, from a spatially homogeneous (disordered) phase to a charge segregated one, where the excluded volume constraint leads to the mutual blocking of particles. We will focus on the disordered phase of the system, where we have a sound analytic understanding of the dynamics in terms of Langevin equations. Performing Monte Carlo simulations and measuring the full distributions of several structure factors, we find that the Gaussian approximation is extremely effective in predicting these properties of our non-equilibrium system. In contrast to the earlier study [8], the structure factors here form a $2 \times 2$ matrix, due to the presence of two species of particles, and the real and imaginary parts of the off-diagonal elements exhibit a characteristic asymmetry. The measurement of these distributions can be used to extract considerable information about the structure of the underlying linearized equations of motion, independent of model details. 


\section{THE MICROSCOPIC MODEL AND THE SIMULATIONS}

We consider a two dimensional fully periodic lattice with $L \times L$ sites, each of which can be empty or occupied by a single particle. Since there are two species we need two occupation variables $n_{\mathbf{x}}^{+}$and $n_{\mathbf{x}}^{-}$, with $n$ being 0 or 1 , depending on whether a positive or negative particle is present at site $\mathbf{x}$. Although we refer to these particles as "charged", they do not interact via the Coulomb potential. Instead, they respond to an external, uniform "electric" field $E$, directed along a specific lattice axis which we label the $+x_{\|}$-direction. We restrict ourselves to zero total charge, i.e., $\sum_{\mathbf{x}}\left[n_{\mathbf{x}}^{+}-n_{\mathbf{x}}^{-}\right]=0$. In the absence of the drive, the dynamics does not distinguish between the different species: both types hop randomly to nearest neighbor empty sites, with a rate $\Gamma$. The electric field introduces a bias into these rates in such a way that jumps against the force will be exponentially suppressed. During one Monte Carlo step $2 L^{2}$ nearest neighbor bonds are chosen randomly. If a particle-hole pair is encountered, an exchange takes place with probability $W=\Gamma \min \left\{1, \exp \left(q E \delta x_{\|}\right)\right\}$, where $q= \pm 1$ is the charge of the particle and $\delta x_{\|}= \pm 1,0$ is the change of the $x_{\|}$coordinate of the particle due to the jump.

For our simulations, we set $\Gamma=1$. Using $60 \times 60$ lattices, we initialize the system with random configurations of various particle densities and carry out runs ranging from 2.5 to $5 \times 10^{5}$ MCS. After allowing 62500 MCS for the system to settle into a steady state, we measure the Fourier transforms of $n_{\mathbf{x}}^{ \pm}$every 125 MCS, defining them in the usual way:

$$
n_{\mathbf{k}}^{ \pm}=\sum_{\mathbf{x}} e^{-i \mathbf{k x}} n_{\mathbf{x}}^{ \pm}
$$

In the literature, the term "structure factor" typically refers to the (ensemble- or time-) average of density-density operators in momentum space, i.e.,

$$
S^{\alpha \beta}(\mathbf{k}) \equiv \frac{1}{V}\left\langle n_{\mathbf{k}}^{\alpha} n_{-\mathbf{k}}^{\beta}\right\rangle,
$$

where $\alpha, \beta=+,-; \mathbf{k}=\frac{2 \pi}{L}\left(m_{\perp}, m_{\|}\right)$and $V=L^{2}$ is the volume. Instead of the overall averages, we construct histograms for $\frac{n_{\mathbf{k}}^{+} n_{-\mathbf{k}}^{+}}{V}, \frac{\operatorname{Re}\left[n_{\mathbf{k}}^{+} n_{-\mathbf{k}}^{-}\right]}{V}$ and $\frac{\operatorname{Im}\left[n_{\mathbf{k}}^{+} n_{-\mathbf{k}}^{-}\right]}{V}$ from their time series in the steady state. We will use the somewhat loose term "structure factors" for these fluctuating quantities themselves. By symmetry, $\frac{n_{\mathbf{k}}^{+} n_{-\mathbf{k}}^{+}}{V}$ and $\frac{n_{\mathbf{k}}^{-} n_{-\mathbf{k}}^{-}}{V}$ are distributed identically, and we consider only the former. The results are presented in Figure 1 and 2 for the smallest longitudinal and transverse wave vectors. Before discussing the data, we will first present the theoretical framework within which they can be understood.

\section{COARSE-GRAINED DESCRIPTION}

Being interested in the large distance (small $\mathbf{k}$ ) behavior, we may exploit the simplest approach, i.e., to use coarsegrained equations of motions for the slowly varying local densities $\varrho^{ \pm}(\mathbf{x}, t)$. These can be considered as the naive continuum limits of the average lattice occupation numbers $\left\langle n_{\mathbf{x}}^{ \pm}\right\rangle$[] $]$]. For generality, we consider the d-dimensional case when $\mathbf{x}_{\|}$is directed along the electric field and $\mathbf{x}_{\perp}$ lies in the $d-1$ dimensional subspace transverse to the field. Then the mean-field equations of motion for the charge densities follow as:

$$
\partial_{t} \varrho^{ \pm}=-\nabla \boldsymbol{\Gamma}\left\{\varrho^{ \pm} \stackrel{\leftrightarrow}{\nabla}\left(1-\varrho^{+}-\varrho^{-}\right) \pm \varepsilon \hat{\mathbf{x}}_{\|} \varrho^{ \pm}\left(1-\varrho^{+}-\varrho^{-}\right)\right\}
$$

Here, $\boldsymbol{\Gamma}$ is the diffusion matrix which is diagonal but not a simple multiple of the unit matrix, due to the anisotropies induced by the bias. $\stackrel{\leftrightarrow}{\nabla}$ is the asymmetric gradient operator, acting on any two functions $f$ and $g$ according to $f \stackrel{\leftrightarrow}{\nabla} g=f \nabla g-g \nabla f . \varepsilon$ is the coarse-grained bias and $\hat{\mathbf{x}}_{\|}$is the unit vector along the $x_{\|}$direction. The coefficients appearing in Eqn. (3) are functions of the microscopic control parameters; however, their precise dependence need not be known.

To study small fluctuations in the disordered phase, we linearize (3) about the homogeneous time-independent solution $\bar{\varrho}$ and add conserved noise terms, to model the fast degrees of freedom. Writing $\varrho^{ \pm}(\mathbf{x}, t)=\bar{\varrho}+\chi^{ \pm}(\mathbf{x}, t)$, we arrive at the Langevin equations for the density fluctuations:

$$
\partial_{t} \chi^{\alpha}(\mathbf{x}, t)=\mathcal{L}^{\alpha \beta}(\boldsymbol{\nabla}) \chi^{\beta}(\mathbf{x}, t)-\nabla \boldsymbol{\eta}^{\alpha}(\mathbf{x}, t),
$$

where 


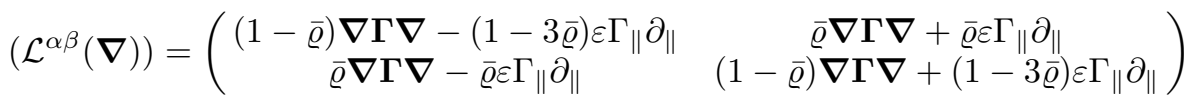

and summation over repeated indices is implied in (值) and in the following. Here, $\boldsymbol{\eta}^{ \pm}(\mathbf{x}, t)$ are Gaussian white noise terms, satisfying:

$$
\begin{aligned}
\left\langle\eta_{i}^{\alpha}(\mathbf{x}, t)\right\rangle & =0 \\
\left\langle\eta_{i}^{\alpha}(\mathbf{x}, t) \eta_{j}^{\beta}\left(\mathbf{x}^{\prime}, t^{\prime}\right)\right\rangle & =2 \delta^{\alpha \beta} \sigma_{i j} \delta\left(\mathbf{x}-\mathbf{x}^{\prime}\right) \delta\left(t-t^{\prime}\right),
\end{aligned}
$$

where $\alpha, \beta=+,-; i, j=1,2, \ldots d$ and $\delta^{\alpha \beta}$ is the Kronecker-symbol. $\left(\sigma_{i j}\right)=\boldsymbol{\sigma}$ is the noise matrix, and similar to $\boldsymbol{\Gamma}$, it is diagonal but not proportional to the unit matrix, due to the bias. To describe systems in thermal equilibrium, the fluctuation-dissipation theorem would require $\boldsymbol{\sigma} \propto \boldsymbol{\Gamma}$. In particular, in the absence of the drive, we would have $\boldsymbol{\sigma}=\bar{\rho}(1-2 \bar{\rho}) \boldsymbol{\Gamma}$ here. However, when driven, the proportionality is not expected to hold, since the diffusion and noise matrices are renormalized differently by the drive $\varepsilon$, similar to the driven single species case 㺻. Finally, we point out that $\boldsymbol{\eta}^{+}$and $\boldsymbol{\eta}^{-}$are uncorrelated, due to the fact that charge exchange is not allowed.

Introducing the Fourier transforms for the density fluctuations (and similarly for the noise)

$$
\chi^{ \pm}(\mathbf{k}, \omega)=\int d t d^{d} x \chi^{ \pm}(\mathbf{x}, t) e^{-i(\omega t+\mathbf{k x})},
$$

the solution to (1) is trivial:

$$
\chi^{\alpha}(\mathbf{k}, \omega)=\left(L^{-1}\right)^{\alpha \beta} i \mathbf{k} \boldsymbol{\eta}^{\beta}(\mathbf{k}, \omega),
$$

where $L^{\alpha \beta}=\mathcal{L}^{\alpha \beta}(i \mathbf{k})-i \omega \delta^{\alpha \beta}$. According to the definition of the full dynamic structure factors

$$
S^{\alpha \beta}(\mathbf{k}, \omega)\left[(2 \pi)^{d+1} \delta\left(\mathbf{k}+\mathbf{k}^{\prime}\right) \delta\left(\omega+\omega^{\prime}\right)\right] \equiv\left\langle\chi^{\alpha}(\mathbf{k}, \omega) \chi^{\beta}\left(\mathbf{k}^{\prime}, \omega^{\prime}\right)\right\rangle,
$$

we can integrate them over $\omega$ to obtain the steady state structure factors:

$$
S^{\alpha \beta}(\mathbf{k})\left[(2 \pi)^{d} \delta\left(\mathbf{k}+\mathbf{k}^{\prime}\right)\right] \equiv\left\langle\chi^{\alpha}(\mathbf{k}, t) \chi^{\beta}\left(\mathbf{k}^{\prime}, t\right)\right\rangle .
$$

Note that they are just the Fourier transforms of the usual equal-time correlation functions $\left\langle\chi^{\alpha}(\mathbf{x}, t) \chi^{\beta}(\mathbf{0}, t)\right\rangle \equiv$ $G^{\alpha \beta}(\mathbf{x})$. The explicit forms of the structure factors are:

$$
\begin{aligned}
S^{++}(\mathbf{k}) & =\frac{(1-\bar{\varrho})}{(1-2 \bar{\varrho})} \frac{\mathbf{k} \boldsymbol{\sigma} \mathbf{k}}{\mathbf{k} \boldsymbol{\Gamma} \mathbf{k}} \frac{(\mathbf{k} \boldsymbol{\Gamma k})^{2}+\frac{(1-3 \bar{\varrho})^{2}}{(1-\bar{\varrho})^{2}} \Gamma_{\|}^{2} \varepsilon^{2} k_{\|}^{2}}{(\mathbf{k} \mathbf{k})^{2}+(1-4 \bar{\varrho}) \Gamma_{\|}^{2} \varepsilon^{2} k_{\|}^{2}} \\
\operatorname{Re}\left\{S^{+-}(\mathbf{k})\right\} & =-\frac{\bar{\varrho}}{(1-2 \bar{\varrho})} \frac{\mathbf{k} \boldsymbol{\sigma} \mathbf{k}}{\mathbf{k} \boldsymbol{\Gamma} \mathbf{k}} \frac{(\mathbf{k} \boldsymbol{\Gamma} \mathbf{k})^{2}-\frac{(1-3 \bar{\varrho})}{(1-\bar{\varrho})} \Gamma_{\|}^{2} \varepsilon^{2} k_{\|}^{2}}{(\mathbf{k} \mathbf{k})^{2}+(1-4 \bar{\varrho}) \Gamma_{\|}^{2} \varepsilon^{2} k_{\|}^{2}} \\
\operatorname{Im}\left\{S^{+-}(\mathbf{k})\right\} & =\frac{2 \bar{\varrho}}{(1-\bar{\varrho})} \frac{\mathbf{k} \boldsymbol{\sigma} \mathbf{k} \Gamma_{\|} \varepsilon k_{\|}}{(\mathbf{k} \boldsymbol{\Gamma} \mathbf{k})^{2}+(1-4 \varrho) \Gamma_{\|}^{2} \varepsilon^{2} k_{\|}^{2}},
\end{aligned}
$$

where $\mathbf{k}=\left(\mathbf{k}_{\perp}, k_{\|}\right)$. They clearly exhibit a finite discontinuity singularity at the origin, i.e., $\lim _{k_{\|} \rightarrow 0} S^{\alpha \beta}\left(\mathbf{0}, k_{\|}\right) \neq$ $\lim _{\mathbf{k}_{\perp} \rightarrow \mathbf{0}} S^{\alpha \beta}\left(\mathbf{k}_{\perp}, 0\right)$ which translates to power law decays in the spatial correlations. In particular, we find the expected [17] power law, $r^{-d}$, related to $\boldsymbol{\sigma} \not \subset \boldsymbol{\Gamma}$, typical of non-equilibrium steady states of a system with anisotropy and subjected to a conservation law. In addition, a novel power, $r^{-(d+1) / 2}[7]$, controls correlations along the bias direction.

Next, we seek the full probability distributions of the density-density operators. Thus, for each $\mathbf{k}$ vector, we construct the distributions separately for $\frac{\chi^{+}(\mathbf{k}, t) \chi^{+}(-\mathbf{k}, t)}{V}, \frac{\operatorname{Re}\left[\chi^{+}(\mathbf{k}, t) \chi^{-}(-\mathbf{k}, t)\right]}{V}$ and $\frac{\operatorname{Im}\left[\chi^{+}(\mathbf{k}, t) \chi^{-}(-\mathbf{k}, t)\right]}{V}$, represented by $s^{++}, s_{r}^{+-}$ and $s_{i}^{+-}$:

$$
\begin{aligned}
& P^{++}\left(s^{++} ; \mathbf{k}\right)=\left\langle\delta\left(\frac{\chi^{+}(\mathbf{k}, t) \chi^{+^{*}}(\mathbf{k}, t)}{V}-s^{++}\right)\right\rangle \\
& P_{r}^{+-}\left(s_{r}^{+-} ; \mathbf{k}\right)=\left\langle\delta\left(\frac{\operatorname{Re}\left[\chi^{+}(\mathbf{k}, t) \chi^{-*}(\mathbf{k}, t)\right]}{V}-s_{r}^{+-}\right)\right\rangle \\
& P_{i}^{+-}\left(s_{i}^{+-} ; \mathbf{k}\right)=\left\langle\delta\left(\frac{\operatorname{Im}\left[\chi^{+}(\mathbf{k}, t) \chi^{-*}(\mathbf{k}, t)\right]}{V}-s_{i}^{+-}\right)\right\rangle .
\end{aligned}
$$


The normalization by $V$ helps to avoid infinities in the expectation values of $s^{++}, s_{r}^{+-}, s_{i}^{+-}$as can be seen from (10) and by noting that $(2 \pi)^{d} \delta(\mathbf{k}=\mathbf{0})=V$ in the infinite volume limit. Also note that we used $\chi^{ \pm}(-\mathbf{k}, t)=\chi^{ \pm^{*}}(\mathbf{k}, t)$, since the densities $\chi^{ \pm}(\mathbf{r}, t)$ are real. For our purposes, we need both, the density fluctuations and the explicit distribution of the noise, in the $(\mathbf{k}, t)$ domain. Within the linear stability regime of the disordered phase, each matrix element of $\left(\left(L^{-1}\right)^{\alpha \beta}\right)$ has two poles in the positive $\omega$ half-plane corresponding to two stable eigenvalues of $\left(\mathcal{L}^{\alpha \beta}\right)$. Thus, from (8) we find:

$$
\chi^{\alpha}(\mathbf{k}, t)=\int_{-\infty}^{t} d t^{\prime}\left[\int_{-\infty}^{\infty} \frac{d \omega}{2 \pi} e^{i \omega\left(t-t^{\prime}\right)}\left(L^{-1}\right)^{\alpha \beta}\right] i \mathbf{k} \boldsymbol{\eta}^{\beta}\left(\mathbf{k}, t^{\prime}\right)
$$

with

$$
P\left[\eta_{i}^{\alpha}(\mathbf{k}, t)\right] \propto \exp \left\{-\frac{1}{2} \int d t d^{d} k \eta_{i}^{\alpha}(\mathbf{k}, t) \frac{\left(D^{-1}\right)_{i j}^{\alpha \beta}}{(2 \pi)^{d}} \eta_{j}^{\beta^{*}}(\mathbf{k}, t)\right\},
$$

where $D_{i j}^{\alpha \beta} \equiv 2 \delta^{\alpha \beta} \sigma_{i j}$ in our model. Due to translational invariance, fields with different $\mathbf{k}$ vectors are decoupled, so we will suppress $\mathbf{k}$ in the following. Then (13) can be written as

$$
\chi^{\alpha}(t)=v_{j}^{\alpha \beta}\left(t, t^{\prime}\right) \eta_{j}^{\beta}\left(t^{\prime}\right)
$$

where

$$
v_{j}^{\alpha \beta}\left(t, t^{\prime}\right)=\Theta\left(t-t^{\prime}\right)\left[\int_{-\infty}^{\infty} \frac{d \omega}{2 \pi} e^{i \omega\left(t-t^{\prime}\right)}\left(L^{-1}\right)^{\alpha \beta}\right] i k_{j} .
$$

Note that summation over repeated indices also includes an integral over $t^{\prime}$ in (15).

We start with the probability distribution of $\frac{\chi^{+}(t) \chi^{+*}(t)}{V}$ by first finding its characteristic function, i.e.,

$$
\tilde{P}^{++}(\Omega)=\int_{-\infty}^{\infty} d s^{++} e^{i \Omega s^{++}} P^{++}\left(s^{++}\right)=\left\langle e^{i \Omega \frac{\chi^{+}(t) \chi^{+*}(t)}{V}}\right\rangle .
$$

When performing the average in (17), all integrations over the noise are trivial, except those associated with $\pm \mathbf{k}$. Thus, we need to evaluate the following integral:

$$
\tilde{P}^{++}(\Omega)=\int \prod_{t^{\prime}, \gamma, j} d \eta_{j}^{\gamma}\left(t^{\prime}\right) d \eta_{j}^{\gamma^{*}}\left(t^{\prime}\right) P\left[\eta_{i}^{\alpha}(t), \eta_{i}^{\alpha *}(t)\right] e^{i \Omega \frac{\chi^{+}(t) \chi^{+*}(t)}{V}}
$$

where $\eta_{j}^{\gamma}\left(t^{\prime}\right)$ and $\eta_{j}^{\gamma^{*}}\left(t^{\prime}\right)$ are the $\mathbf{k}$ and $-\mathbf{k}$ components of the noise, respectively, yielding the only non-trivial integrations. Inserting (15) into (18), we still have a Gaussian integrand, controlled by the quadratic form:

$$
\eta_{\mu}\left(\left(D^{-1}\right)_{\mu \nu}-i \Omega v_{\mu}^{+} v_{\nu}^{+*}\right) \eta_{\nu}^{*}
$$

where the indices $\mu, \nu$ include all the degrees of freedom left over, namely, time, charge, and spatial component. The corresponding path integrals lead to

$$
\tilde{P}^{++}(\Omega)=\frac{\operatorname{det}\left[\left(D^{-1}\right)_{\mu \nu}\right]}{\operatorname{det}\left[\left(D^{-1}\right)_{\mu \nu}-i \Omega v_{\mu}^{+} v_{\nu}^{+*}\right]}=\frac{1}{\operatorname{det}\left[\delta_{\mu \nu}-i \Omega D_{\mu \gamma} v_{\gamma}^{+} v_{\nu}^{+*}\right]},
$$

where the numerator in the middle expression originates in the normalization factor ensuring $\tilde{P}^{++}(0)=1$. Exploiting the formula $\operatorname{det}\left[\delta_{\mu \nu}+a_{\mu} b_{\nu}\right]=1+a_{\mu} b_{\mu}$, we obtain

$$
\tilde{P}^{++}(\Omega)=\frac{1}{1-i \Omega v_{\mu}^{+} D_{\mu \nu} v_{\nu}^{+*}}
$$

Note that the coefficient of $i \Omega$ is simply the ' ++ ' structure factor:

$$
v_{\mu}^{+} D_{\mu \nu} v_{\nu}^{+^{*}}=\left\langle\frac{\chi^{+}(\mathbf{k}, t) \chi^{+^{*}}(\mathbf{k}, t)}{V}\right\rangle=S^{++}(\mathbf{k}) .
$$


Taking the inverse transform to obtain $P^{++}$, the single pole $-i / S^{++}(\mathbf{k})$ in the lower half $\Omega$-plane yields an exponential distribution for the non-negative variable $s^{++}$, i.e.,

$$
P^{++}\left(s^{++} ; \mathbf{k}\right)=\left\{\begin{array}{cl}
\frac{1}{S^{++}(\mathbf{k})} e^{-s^{++} / S^{++}(\mathbf{k})} & \text { if } s^{++} \geq 0 \\
0 & \text { if } s^{++}<0
\end{array}\right.
$$

We will refer to $1 / S^{++}(\mathbf{k})$ as the "decay factor" of the exponential.

Next, we consider the distribution of $\frac{\operatorname{Re}\left[\chi^{+}(t) \chi^{-*}(t)\right]}{V}$. Following the same steps, we see that the quadratic form controlling the Gaussian integrand is

$$
\eta_{\mu}\left(\left(D^{-1}\right)_{\mu \nu}-\frac{i \Omega}{2}\left(v_{\mu}^{+} v_{\nu}^{-*}+v_{\mu}^{-} v_{\nu}^{+*}\right)\right) \eta_{\nu}^{*}
$$

leading to

$$
\tilde{P}_{r}^{+-}(\Omega)=\frac{1}{\operatorname{det}\left[\delta_{\mu \nu}-\frac{i \Omega}{2}\left(D_{\mu \gamma} v_{\gamma}^{+} v_{\nu}^{-*}+D_{\mu \gamma} v_{\gamma}^{-} v_{\nu}^{+^{*}}\right)\right]} .
$$

Using the formula $\operatorname{det}\left[\delta_{\mu \nu}+a_{\mu} b_{\nu}+c_{\mu} d_{\nu}\right]=1+\left[a_{\mu} b_{\mu}+c_{\mu} d_{\mu}\right]+\left[\left(a_{\mu} b_{\mu}\right)\left(c_{\nu} d_{\nu}\right)-\left(a_{\mu} d_{\mu}\right)\left(b_{\nu} c_{\nu}\right)\right]$ we arrive at

$$
\tilde{P}_{r}^{+-}(\Omega)=\frac{1}{1-i \Omega \operatorname{Re}\left[S^{+-}(\mathbf{k})\right]+\frac{\Omega^{2}}{4}\left[\left|S^{++}(\mathbf{k})\right|^{2}-\left|S^{+-}(\mathbf{k})\right|^{2}\right]},
$$

where, similarly to (22), we have used

$$
v_{\mu}^{+} D_{\mu \nu} v_{\nu}^{-*}=\left\langle\frac{\chi^{+}(\mathbf{k}, t) \chi^{-*}(\mathbf{k}, t)}{V}\right\rangle=S^{+-}(\mathbf{k}) .
$$

Unlike the previous case, $\tilde{P}_{r}^{+-}(\Omega)$ has two poles: one $\left(\Omega_{-}\right)$being on the negative, and the other $\left(\Omega_{+}\right)$on the positive, imaginary axis:

$$
\Omega_{\mp}=\frac{2 i}{\Delta}\left(\operatorname{Re}\left[S^{+-}(\mathbf{k})\right] \mp \sqrt{\Delta+\left(\operatorname{Re}\left[S^{+-}(\mathbf{k})\right]\right)^{2}}\right)
$$

where $\Delta \equiv\left|S^{++}(\mathbf{k})\right|^{2}-\left|S^{+-}(\mathbf{k})\right|^{2}>0$. In general, their magnitudes are different. Thus, the inverse transform yields an asymmetric exponential distribution, characterized by two distinct decay factors $\left|\Omega_{+}\right|$and $\left|\Omega_{-}\right|$:

$$
P_{r}^{+-}\left(s_{r}^{+-} ; \mathbf{k}\right)=\left\{\begin{array}{cl}
\frac{1}{N} e^{-\left|\Omega_{-}\right| s_{r}^{+-}} & \text {if } s_{r}^{+-} \geq 0 \\
\frac{1}{N} e^{\left|\Omega_{+}\right| s_{r}^{+-}} & \text {if } s_{r}^{+-}<0
\end{array}\right.
$$

with $N=\sqrt{\Delta+\left(\operatorname{Re}\left[S^{+-}(\mathbf{k})\right]\right)^{2}}$. To obtain the distribution of $\frac{\operatorname{Im}\left[\chi^{+}(t) \chi^{-*}(t)\right]}{V}$ we simply interchange $\operatorname{Re}\left[S^{+-}(\mathbf{k})\right]$ and $\operatorname{Im}\left[S^{+-}(\mathbf{k})\right]$ in eqs. 28,29$)$.

One important consequence of (23) and (29) is that their standard deviations always take a value greater or equal than the average. In particular

$$
\begin{aligned}
& \sqrt{\overline{\left(s^{++}\right)^{2}}-\left(\overline{s^{++}}\right)^{2}}=S^{++}(\mathbf{k}) \\
& \sqrt{\overline{\left(s_{r}^{+-}\right)^{2}}-\left(\overline{s_{r}^{+-}}\right)^{2}}=\sqrt{\left(\operatorname{Re}\left[S^{+-}(\mathbf{k})\right]\right)^{2}+\frac{\Delta}{2}} \\
& \sqrt{\overline{\left(s_{i}^{+-}\right)^{2}}-\left(\overline{s_{i}^{+-}}\right)^{2}}=\sqrt{\left(\operatorname{Im}\left[S^{+-}(\mathbf{k})\right]\right)^{2}+\frac{\Delta}{2}} .
\end{aligned}
$$

Thus, when measuring structure factors in the disordered phase, fluctuations comparable to the average should not come as a surprise. We also emphasize that formulas (23) and (29) are completely independent of the specific model and can be derived in any multicomponent system. The only necessary conditions are linear Langevin equations with Gaussian noise. 


\section{DISCUSSION AND SUMMARY}

Finally, let us turn to a comparison with the simulation results summarized in Figure 1 and 2 , for the two smallest wave vectors. The ' ++ ' histograms show the usual exponential decay [8], while the ' +- ' histograms clearly represent asymmetric exponential distributions. To test the predictions of our Gaussian theory, namely, that the slopes of the histograms are determined by the structure factor averages themselves, we simply measured these averages, i.e., $S^{++}$ , $\operatorname{Re} S^{+-}$and $\operatorname{Im} S^{+-}$. The ' ++ ' case is particularly simple since the decay factor is just the inverse of $S^{++}$itself. For the two '+-' distributions, we inserted the measured averages into the non-trivial theoretical relationship (28), to find the decay factors $\left|\Omega_{\mp}\right|$. The agreement between simulation results and theory is remarkably good, indicating that linearized Langevin equations are quite acceptable in this regime. While the external field, $E$, may obviously generate renormalizations, these can be absorbed in the effective parameters of the theory, namely the diffusion matrix, the noise matrix, the average density and the coarse-grained bias, leaving the form of the structure factor distributions invariant. However, we must avoid critical fluctuations since the linear approximation will fail to capture their effects correctly.

To summarize, we have analyzed the full structure factor distributions in a simple driven lattice gas. Due to the presence of two species, there are several distinct structure factor distributions here. All are simple exponentials, with the decay factors given in terms of the average structure factors. However, only the "diagonal" elements $P^{++}$and $P^{--}$are characterized by a single decay factor, being distributions for a non-negative variable. In contrast, both $P_{r}^{+-}$ and $P_{i}^{+-}$are combinations of two exponentials, with different decay factors, characterizing positive versus negative arguments, respectively. While this asymmetry may, at first sight, be rather surprising, its origin is quite easily traced to the presence of off-diagonal elements in the matrix $\mathcal{L}^{\alpha \beta}$, Eqn. (5). Thus, the observation of asymmetric structure factor histograms, either in an experiment or a simulation, provides some basic insight into the form of the underlying Langevin equation.

\section{ACKNOWLEDGEMENTS}

We thank Z. Toroczkai, S. Sandow and C. Laberge for many stimulating discussions. This research is supported in part by grants from the National Science Foundation through the Division of Materials Research .

[1] D. Beysens and M. Gbadamassi, Phys. Rev. A22 (1980) 2250; H. Kiefte, M.J. Clouter and R. Penney, Phys. Rev. B30 (1984) 4017; B.M. Law, P.N. Segrè, R.W. Gammon and J.V. Sengers, Phys. Rev. A41 (1990) 816; P.N. Segrè, R.W. Gammon, J.V. Sengers and B.M. Law, Phys. Rev. A45 (1992) 714; P.N Segrè, R. Schmitz and J.V Sengers, Physica A195 (1993) 31.

[2] S. Katz, J.L. Lebowitz and H. Spohn, Phys. Rev. B28 (1983) 1655; J. Stat. Phys. 34 (1984) 497.

[3] B. Schmittmann and R.K.P. Zia, in: Phase Transitions and Critical Phenomena Vol. 17, eds. C. Domb and J.L. Lebowitz, (Academic Press, N.Y., 1995).

[4] H.K. Janssen and B. Schmittmann, Z. Phys. B64 (1986) 503; K.-t Leung and J.L.Cardy, J. Stat. Phys. 44 (1986) 567 and (1986) 1087.

[5] R. Kubo, Rep. Progr. Phys. 29 (1966) 255.

[6] R.K.P. Zia, K. Hwang, B. Schmittmann and K.-t Leung, Physica A194 (1993) 183.

[7] G. Korniss, B. Schmittmann and R.K.P. Zia, Physica A (in press)

[8] M.S. Rudzinsky and R.K.P. Zia, J. Phys. A29 (1996) 6717.

[9] M. Blume, V.J. Emery and R.B. Griffiths, Phys. Rev. A4 (1971) 1071.

[10] R.B. Potts, Proc. Camb. Phil. Soc. 48 (1952) 106; F.Y. Wu, Rev. Mod. Phys. 54 (1982) 235.

[11] B. Schmittmann, K. Hwang and R.K.P. Zia, Europhys. Lett. 19 (1992) 19.

[12] I. Vilfan, R.K.P. Zia and B. Schmittmann, Phys. Rev. Lett. 73 (1994) 2071.

[13] D.P. Foster, C. Godrèche, J. Stat. Phys. 76 (1994) 1129.

[14] G. Korniss, B. Schmittmann and R.K.P. Zia, Europhys. Lett. 32 (1995) 49 and J. Stat. Phys. (Febr., 1997) in press

[15] O. Biham, A.A. Middleton, and D. Levine, Phys. Rev. A46 (1992) R6128; K.-t. Leung, Phys. Rev. Lett. 73 (1994) 2386

[16] M.R. Evans, D.P. Foster, C. Godrèche and D. Mukamel, Phys. Rev. Lett. 78 (1995) 208 and J. Stat. Phys. 80 (1995) 69.

[17] M.Q. Zhang, J.-S. Wang, J.L Lebowitz and J.L. Vallés, J. Stat. Phys. 52 (1988) 1461. 

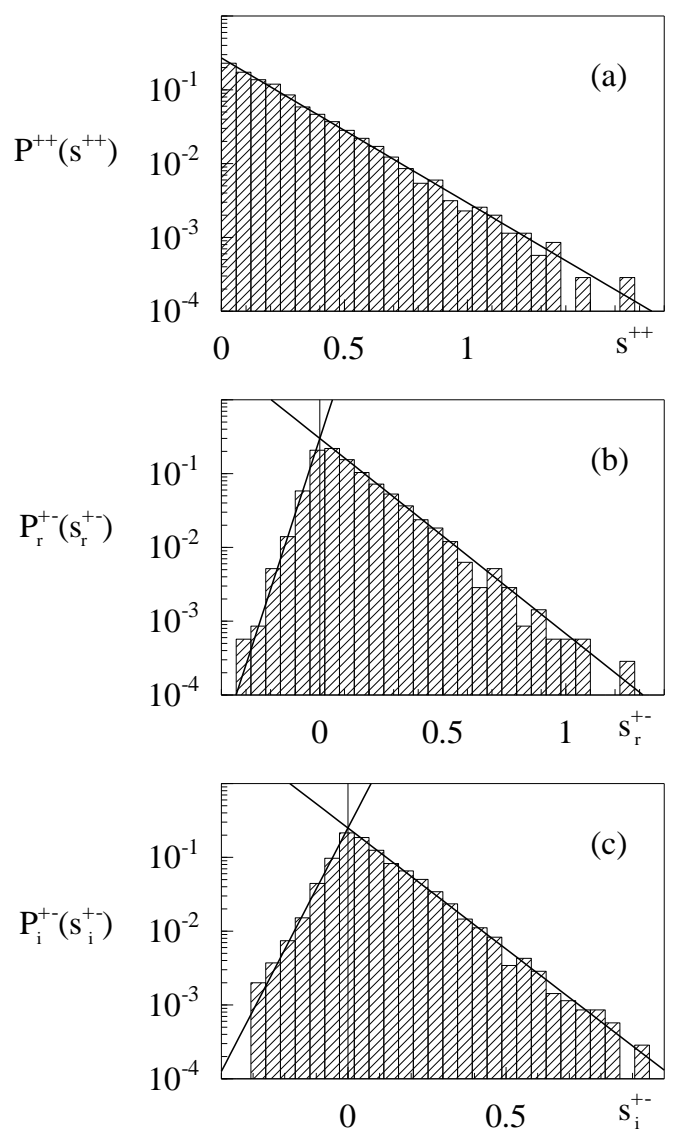

FIG. 1. Histograms representing the distributions of the $\mathbf{k}=\frac{2 \pi}{L}(0,1)$ structure factors for (a) $\frac{n_{\mathbf{k}}^{+} n_{-\mathbf{k}}^{+}}{V}$, (b) $\frac{\operatorname{Re}\left[n_{\mathbf{k}}^{+} n_{-\mathbf{k}}^{-}\right]}{V}$ and (c) $\frac{\operatorname{Im}\left[n_{\mathbf{k}}^{+} n_{-\mathbf{k}}^{-}\right]}{V} . \quad L=60, E=0.471$ and $\varrho=0.175$. Theoretical distributions (a) $P^{++}\left(s^{++} ; \mathbf{k}\right)$, (b) $P_{r}^{+-}\left(s_{r}^{+-} ; \mathbf{k}\right)$ and (c) $P_{i}^{+-}\left(s_{i}^{+-} ; \mathbf{k}\right)$ are plotted with solid lines on the same graphs. 

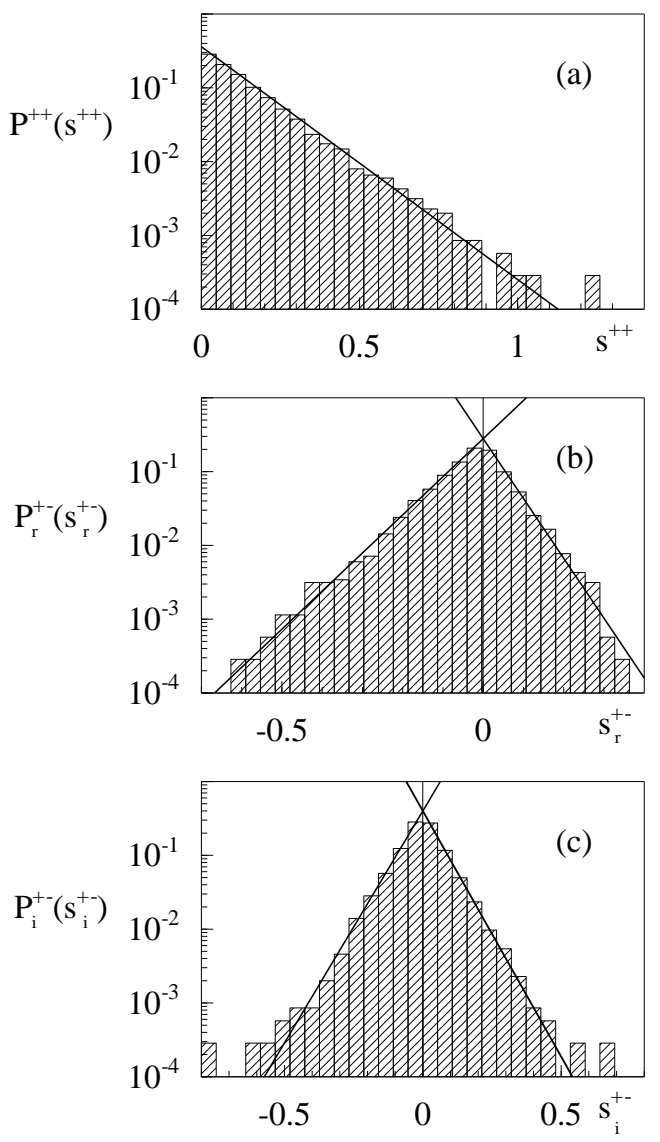

FIG. 2. Histograms representing the distributions of the $\mathbf{k}=\frac{2 \pi}{L}(1,0)$ structure factors for (a) $\frac{n_{\mathbf{k}}^{+} n_{-\mathbf{k}}^{+}}{V}$, (b) $\frac{\operatorname{Re}\left[n_{\mathbf{k}}^{+} n_{-\mathbf{k}}^{-}\right]}{V}$ and (c) $\frac{\operatorname{Im}\left[n_{\mathbf{k}}^{+} n_{-\mathbf{k}}^{-}\right]}{V} . \quad L=60, E=0.471$ and $\bar{\varrho}=0.175$. Theoretical distributions (a) $P^{++}\left(s^{++} ; \mathbf{k}\right)$, (b) $P_{r}^{+-}\left(s_{r}^{+-} ; \mathbf{k}\right)$ and (c) $P_{i}^{+-}\left(s_{i}^{+-} ; \mathbf{k}\right)$ are plotted with solid lines on the same graphs. 